\title{
ESTUDIO DE ACTITUDES HACIA EL ABORTO EN UNIVERSITARIOS DE MORELOS, MÉXICO
}

\section{STUDY OF ATTITUDES TOWARDS THE ABORTION IN COLLEGE STUDENTS OF MORELOS, MEXICO}

Cruz García Lirios

Psicólogo egresado de la Universidad Autónoma Metropolitana, maestrante en Trabajo Social por la Universidad Nacional Autónoma de México. Autor del libro: "Hidroexclusión. Análisis de los factores psicosociales que impiden la sustentabilidad hídrica". Ha publicado artículos y presentado diversas ponencias sobre Desarrollo Sustentable, Brecha Digital y Exclusión Social. Adscrito al Colegio Tecnológico de México y a la Academia Mexicana de Investigación en

Trabajo Social. garcialirios@gmail.com

\section{Resumen}

El objetivo del presente estudio es demostrar la emergencia psicosocial de las actitudes hacia el aborto inducido. Para tal propósito, se llevó a cabo un estudio en el que se estableció la validez y confiabilidad de una escala actitudinal que mide la propensión y la aversión al futuro personal y familiar en una situación de aborto inducido. El análisis de las evaluaciones actitudinales permitirá diseñar estudios que corroboren la hipótesis en torno a la cual las actitudes hacia el aborto inducido son ambivalentes puesto que coexisten tanto la planificación del futuro personal-familiar con la aversión a dicha situación prospectiva. A la luz de la Teoría de la Acción Razonada y la Teoría del Comportamiento Planificado los resultados develan la relevancia de la problemática abortiva puesto que el crecimiento poblacional y las crisis económicas podrían incentivar la emergencia psicosocial de las actitudes hacia el aborto inducido como un instrumento de planificación o supervivencia familiar. 
Palabras claves: aborto inducido, actitud, evaluación, propensión al futuro y aversión prospectiva.

\section{Abstract}

The objective of the present study is to demonstrate the psycho-social emergency of the attitudes towards the induced abortion. For such intention, it carried out a study in which the validity settled down and trustworthiness of attitude scale that measures the propensity and the personal and the future familiar aversion to in a situation of induced abortion. The analysis of the attitude evaluations will allow designing studies that corroborate the hypothesis around which the attitudes towards the induced abortion are ambivalent since they as much coexist the planning of the future personnel-relative with the aversion to this prospective situation. In the light of the Theory of the Reasoned Action and the Theory of the Planned Behavior the results reveal the problematic relevance of the abortion since the population growth and the economic crises could stimulate the psycho-social emergency of the attitudes towards the abortion induced like a planning family instrument or survival.

Keywords: prospective aversion and, induced abortion attitude evaluation propensity the future. 
La comisión Nacional de Población (Conapo) en su Encuesta Nacional de la Dinámica Demográfica (Enadid) correspondiente al año 2009, observa un mayor porcentaje de mortinato en referencia al aborto entre mujeres de 15 a 49 años.

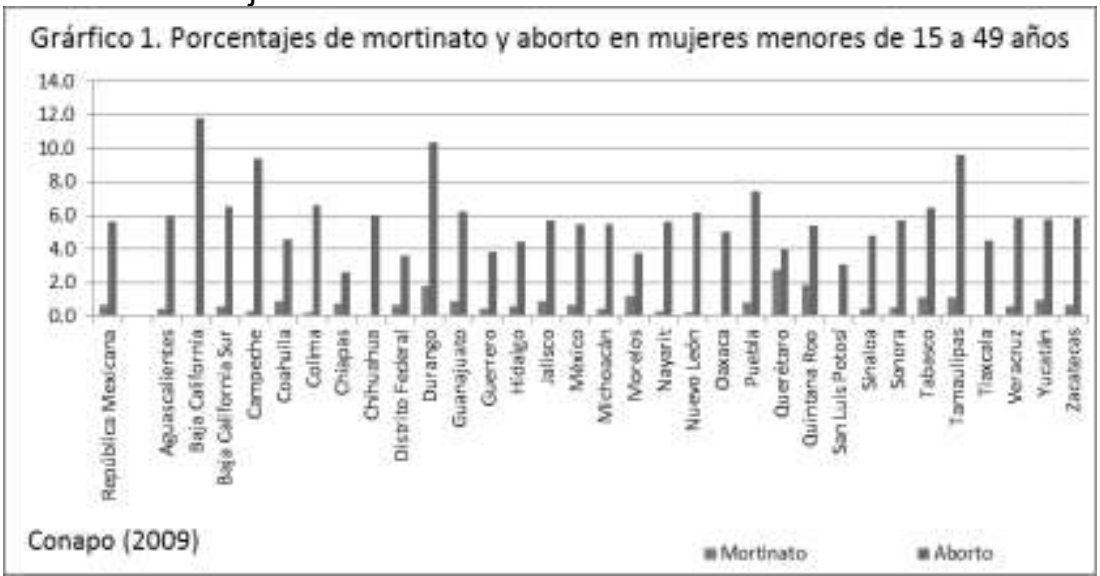

El gráfico 1 muestra una mayor tendencia porcentual de aborto en el estado de Querétaro comparada con la tendencia de aborto en los demás estados que configuran al país. En el caso de la Ciudad de México, donde el aborto asistido es legal, su tendencia porcentual es sólo superior a Aguas Calientes, Baja California Sur, Campeche, Colima, Chihuahua, Guerrero, Michoacán, Nayarit, Nuevo León, Oaxaca, San Luis Potosí, Sinaloa, y Sonora y Tlaxcala. 


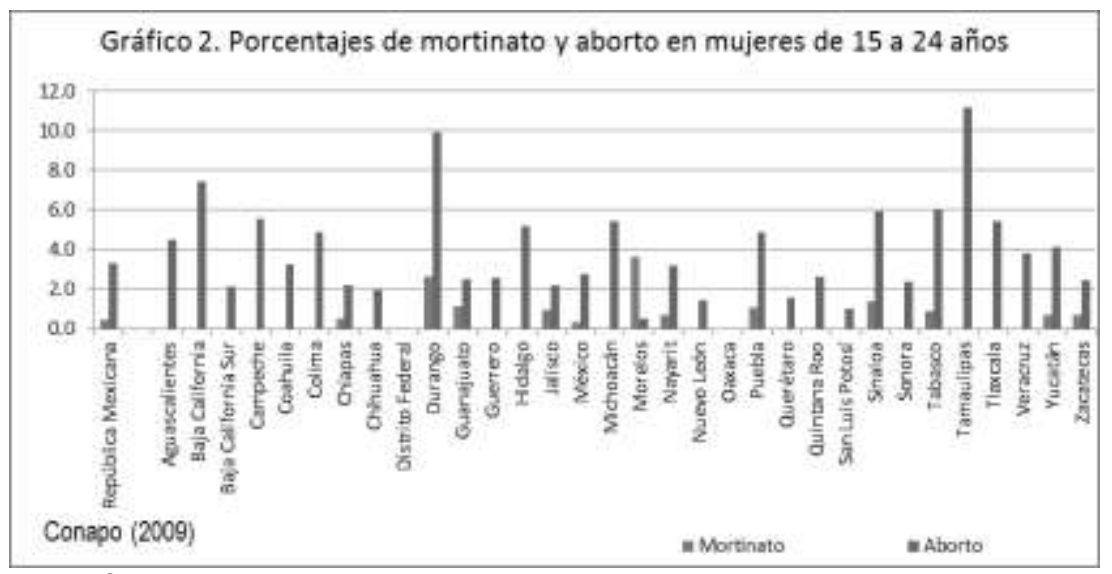

El gráfico 2 muestra una tendencia porcentual mayor en el estado de Morelos entre las mujeres de 15 a 24 años con respecto a los demás estado del país. En el caso de la Ciudad de México, es posible observar una menor tendencia porcentual con respecto a Chiapas, Durango, Guanajuato, Jalisco, Morelos, Nayarit, Puebla, Sinaloa, Tabasco, Yucatán y Zacatecas. Esta tendencia porcentual de aborto, se observa en el gráfico 3 con respecto a las mujeres cuya edad oscila entre los 25 y los 34 años de edad. En este rango de edad, la Ciudad de México tiene una tendencia abortiva mínima. Querétaro ocupa el primer sitio en referencia a los 31 estados que configuran el país.

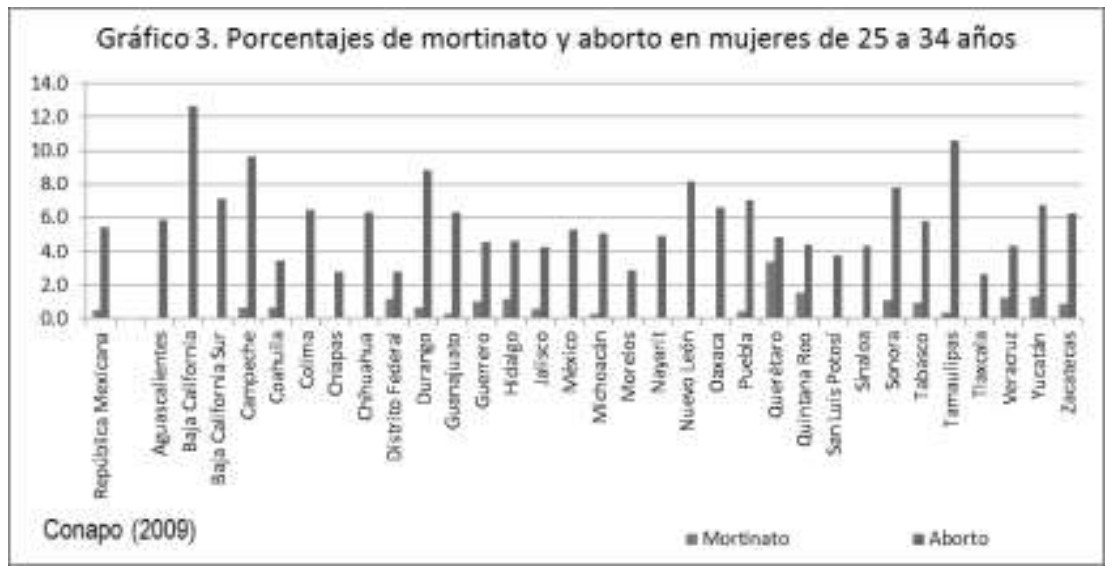


Finalmente, si se consideran los datos relativos a las mujeres entre los 35 y los 49 años, rango de mayor riesgo, Quintana Roo sobresale como el estado con mayor tendencia abortiva y la Ciudad de México ocupa los primeros sitios de la práctica abortiva.

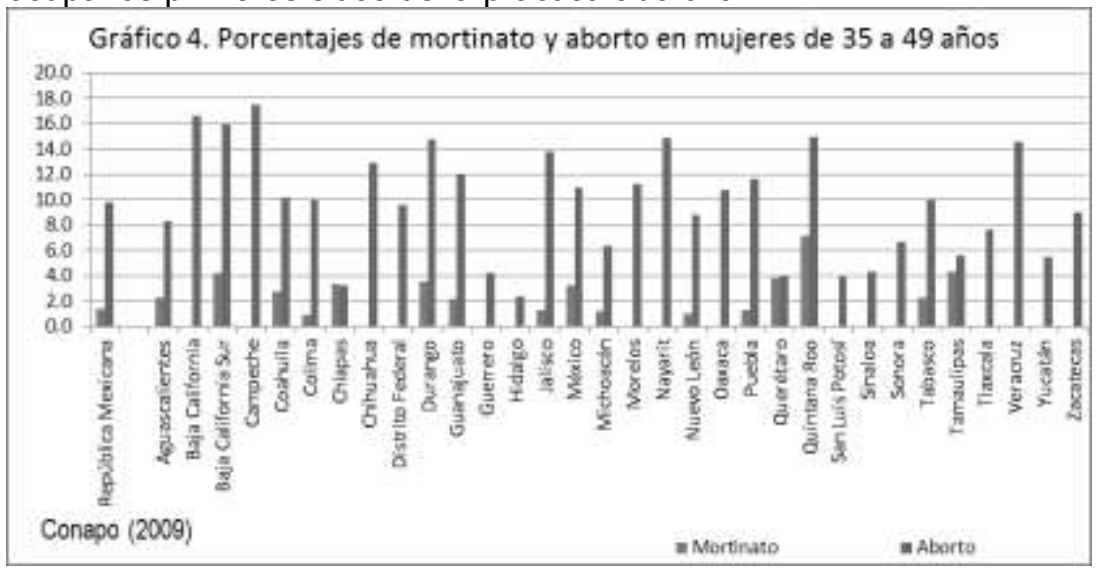

En el caso del estado de Morelos, la práctica abortiva es mínima con respecto a los demás estado de la república mexicana.

En síntesis, la tendencia abortiva identifica a la Ciudad de México, en donde se legisló legalización del aborto, como una entidad intermedia en la práctica abortiva. Querétaro ocupa el primer sitio y el estado de Morelos, a pesar de su cercanía con ambas entidades, ocupa una tendencia abortiva intermedia.

Debido a que la práctica abortiva parece obedecer a una tendencia discontinua y muchas veces contradictoria, es menester analizar los factores psicológicos que facilitan o impiden la decisión de abortar puesto que en la mayoría de los casos, la mujer ejerce el derecho de aborto.

\section{Teorías actitudinales}

En psicología social, el estudio de las actitudes se esgrime a partir de su relación con otras variables de orden cognitivo en relación directa con el comportamiento. En torno a las actitudes, tres modelos las incorporan como variables mediadoras de los efectos culturales tales como las creencias y los valores. 
La Teoría de la Acción Razonada (TRA por sus siglas en inglés), incluye a las actitudes como un factor de expectativas en torno al comportamiento y evaluaciones de las consecuencias de las acciones. La figura 1 muestra la relación causal entre las variables normativas, evaluativas y motivacionales como causas exógenas a la cognición individual, pero en relación causal indirecta con el comportamiento deliberado. Esto significa que las personas llevan a cabo comportamientos que están influidos por su experiencia previa con el objeto actitudinal. En el caso del aborto, la TRA plantearía que la práctica abortiva depende de un cálculo de beneficios y costos más expectativas del futuro a partir de experiencias previas con casos o situaciones de aborto. En este sistema de creencias, las actitudes pueden favorecer o inhibir al sistema de creencias puesto que en tanto disposición general, inciden mínimamente en las decisiones específicas de aborto y las acciones correspondientes (Ajzen, 2001).

Figura 1. Modelo de Acción Razonada

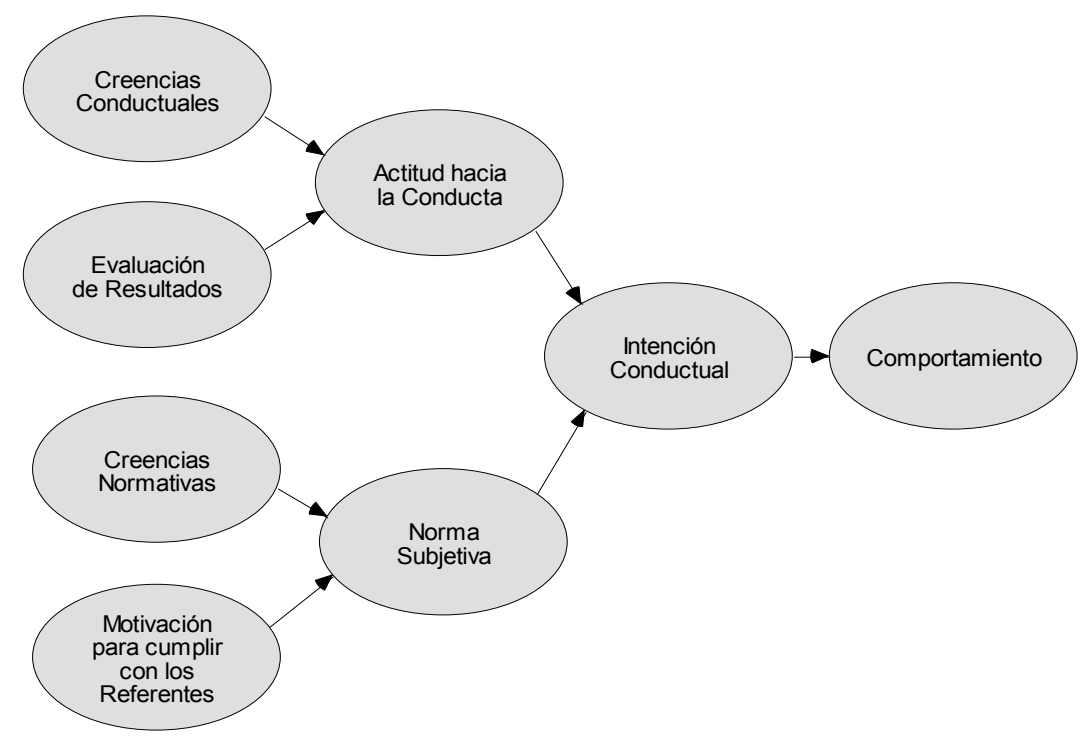

Fuente: Ajzen y Fishbein (1974) 
No obstante, el poder predictivo de la TRA en torno a la práctica abortiva, la relación entre el sistema de creencias y las disposiciones parecen no tener relación. Es decir, si las personas están inmersas en una cultura que considera al aborto como un tabú y una mujer se ha embarazado sin desearlo, entonces sus disposiciones podrían no tener relación con sus creencias. La situación de embarazo no deseado tendría una mayor influencia que el tabú de la cultura respecto a las madres solteras. Este caso llevó a uno de los autores de la TRA a perfeccionar el modelo al que llamó teoría del Comportamiento Planificado (TPB por sus siglas en inglés).

La figura 2 muestra el modelo de la TPB. Pueden observarse: un sistema de creencias que incide sobre el comportamiento a través de actitudes, normas, percepciones e intenciones. A la luz de la TPB, el aborto es el resultado de una cadena de razonamientos cuyo objetivo es maximizar las ganancias y minimizar las pérdidas. Ante la decisión de abortar un embarazo no deseado, se encuentran tres creencias básicas sobre la práctica abortiva, la norma personal y la percepción de control de la situación. El acto de abortar depende de lo que la persona cree que le depara el futuro y su capacidad de autocontrol de la situación. Una vez que se ha equilibrado el sistema de creencias generales con las cogniciones individuales, los avances en torno a la predicción de la práctica abortiva se rediseñaron en un tercer modelo conocido como Teoría del Comportamiento Improvisado (TCl).

Figura 2. Modelo del Comportamiento Planificado 


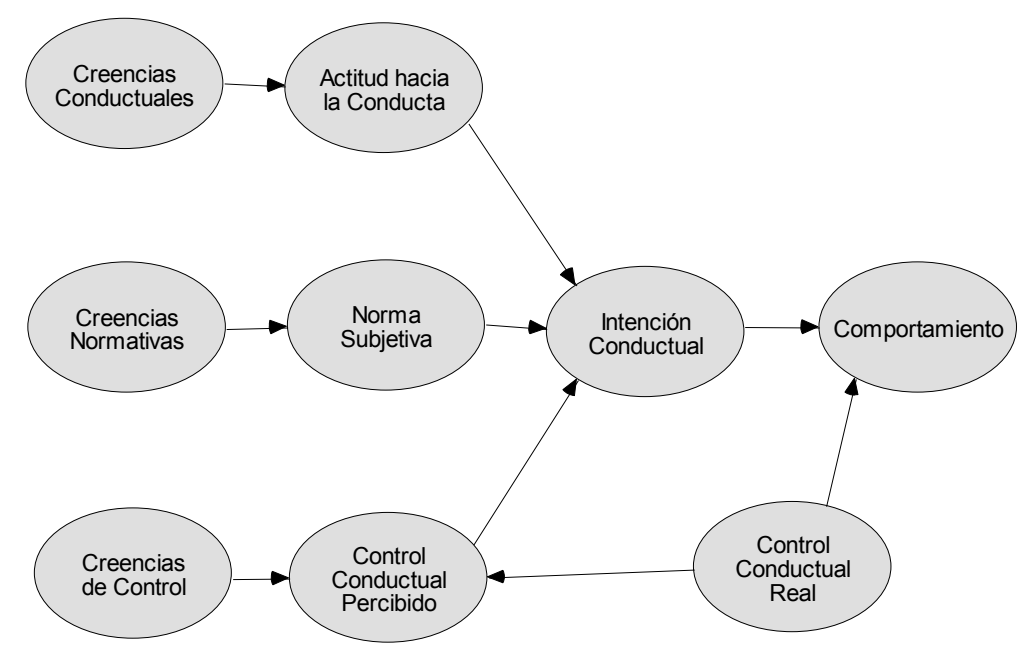

Fuente: Ajzen (1991)

A diferencia de la TRA y TPB el TCl excluye al sistema de creencias e incorpora variables de orden emocional-afectivo más que cognitivo y racional. Es decir, la actitud ya no es una intermediaria entre los sistemas de creencias, deliberados, planificados y sistemáticos y la práctica abortiva. La figura 3 muestra al entorno del individuo como determinante exógeno de sus comportamientos respecto al aborto.

Se trata de un modelo en el que la práctica abortiva se explica a partir de un microsistema favorable a la práctica abortiva. No obstante, la $\mathrm{TCl}$ es un modelo ecológico que trata de establecer la relación causal entre los componentes.

Figura 3. Modelo del Comportamiento Improvisado 


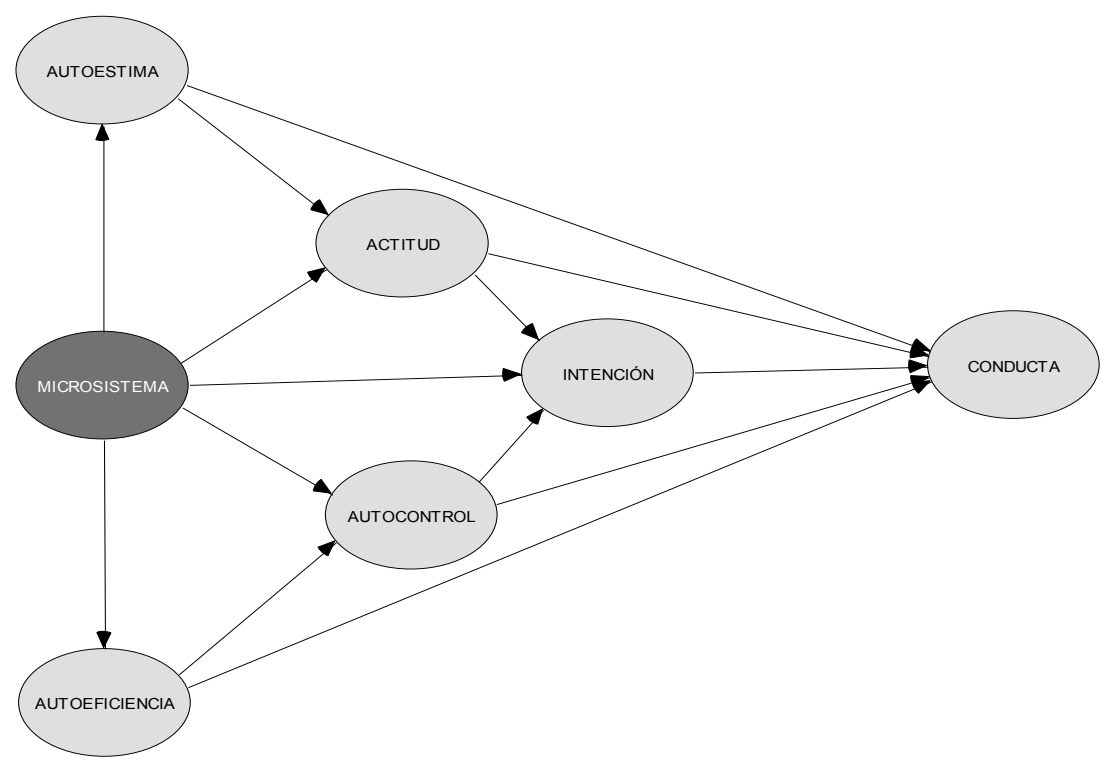

Un incremento en los valores de la autoestima y la autoeficiencia propicia un aumento en la actitud, el autocontrol y la intención sobre el aborto. Es decir, las mujeres beneficiadas con la Ley de Aborto Legal Asistido, tendrían una autoestima que incentiva su disposición de llevarse a cabo un aborto y que al final terminaran solicitando el derecho a interrumpir su embarazo. Este proceso implica razonamientos heurísticos en torno a los cuales la actitud se configura a favor o en contra de la ley, de quienes asumen su derecho, de quienes asisten la interrupción y de quienes promueven la práctica abortiva.

En tal sentido, es menester revisar los estudios de actitudes hacia el aborto, su asistencia médica, su promoción y el sistema de creencias en torno a la práctica abortiva.

\section{Estudios actitudinales del aborto}

El estado del arte define al aborto inducido como el procedimiento realizado para terminar un embarazo no deseado practicado por personas que carecen de las calificaciones necesarias o practicado en un ambiente en las mínimas condiciones de seguridad médicas o de ambos (Briozzo, Vidiella, Vidarte, Pons y Cuadro, 2002). Principalmente, el aborto inducido se lleva a cabo en lugares 
socioeconómicamente vulnerables, marginales o excluidos en los que la población más joven o adolescente presenta una tasa superior en comparación con los demás estratos económicos y poblaciones (González, 2000: p. 72). Respecto a los conocimientos y las actitudes hacia la práctica abortiva inducida, se observa una tendencia conservadora respecto a la responsabilidad de practicarse un aborto. En este sentido, la situación económica y la presión conyugal o familiar son determinantes del aborto inducido.

Lara (1987: p. 30) demostró diferencias entre hombres y mujeres respecto al aborto inducido. El 50 por ciento de las mujeres y el 18 por ciento de los hombres aceptaron la práctica abortiva inducida. El mismo autor encontró diferencias significativas con respecto al ámbito de trabajo.

Cabezas, Langer, Álvarez y Bustamant (1998: p. 268) establecieron diferencias significativas entre las características sociodemográficas y el aborto inducido. Respecto a la edad, encontraron diferencias entre mujeres menores de 20 años, entre 20 y 25 años y mayores de 25 años con respecto al aborto inducido. En torno al nivel de estudios, establecieron diferencias significativas entre quienes terminaron la primaria, secundaria, preparatoria y universidad con respecto al aborto inducido. El estado civil también arrojó diferencias significativas entre casadas, solteras y en unión libre con respecto al aborto provocado. Respecto a la ocupación, también se encontraron diferencias significativas entre quienes son profesionistas, obreras, estudiantes o amas de casa en torno al aborto practicado. Finalmente, la edad al momento de tener una relación sexual; menos de 20 , entre 20 y 24 y más de 24 años, también influyó en la práctica abortiva. Sólo en el tipo de raza; blanca, negra y mestiza no observaron diferencias significativas.

Sánchez, Jiménez y merino (1999: p. 10) encontraron una actitud positiva hacia el aborto legal asistido y la práctica de aborto inducido o asistido por personal médico de alguna institución o dependencia de salud. Establecieron diferencias significativas por disciplinas de conocimiento con respecto a la práctica abortiva inducida.

Galváo, et, al. (2000: p. 5) encontraron que 75.4 por ciento del personal médico recetó un método de anticoncepción de emergencia en la muestra de especialistas encuestados. 42.8 recetaron la píldora de anticoncepción de emergencia en una situación de riesgo.

Ramírez (2000: p. 50) encontró que el 22 por ciento de los hombres encuestados consideraron al aborto como un tema esencial de la 
sexualidad. En contraste, el 77 por ciento de las mujeres identificaron al aborto como la principal problemática en torno a su sexualidad.

Castro, et, al. (2001: págs. 23 a 25) encontraron que el 8 por ciento de la muestra encuestada no conocía los métodos de anticoncepción de emergencia, 84.9 por ciento declararon tratamientos hormonales, 69.7 por ciento citaron como efecto secundario los vómitos, el 49.6 por ciento declararon que si la anticoncepción la solicitaban menores de edad deberían ir acompañados de algún familiar mayor.

García, Lara y Goldman (2003: págs. 98 y 99) encontraron que el 54 por ciento de la muestra encuestada, considera que la opinión de las mujeres debe ser escuchada en torno a la práctica abortiva inducida. El 34 por ciento cree que las mujeres abortan por irresponsables, el 56 por ciento considera que las instituciones de salud deben ofrecer la asistencia de aborto hasta el primer trimestre de gestación. Finalmente, el 85 por ciento considera que el aborto inducido está justificado si la mujer corre un gran peligro de salud.

Salazar (2007: p. 42) encontró que las actitudes hacia el aborto son semiliberales con un 66.2 por ciento, una actitud semiconservadora de 26.2 por ciento. En promedio, la muestra tuvo su primera experiencia sexual a los 19 años misma edad en la que consideran que prefieren utilizar algún preservativo para evitar embarazos no deseados.

Tapia, Villaseñor y Nuño (2008: p. 40) encontraron una actitud favorable hacia la anticoncepción de emergencia. El 95 por ciento de la muestra declaró haber escuchado el método de anticoncepción de emergencia y el 80 por ciento lo consideró un método adecuado.

Tavara y Sacsa (2008: p. 14) establecieron diferencias significativas entre las características sociodemográficas y los conocimientos sobre el aborto inducido. Edad, estado civil y experiencia laboral fueron estadísticamente significativas en relación al conocimiento abortivo inducido. El legrado uterino, fue la técnica y el método abortivo mayormente mencionado en las entrevistas.

Calderón y Alzamora (2009: p. 112) encontraron una relación directa entre el consumo de licor entre las amistades de las mujeres que abortaban. El 65 por ciento de la muestra de mujeres abortivas tenía una amistad que consumía frecuentemente alcohol y el 41 por ciento de las mujeres casadas tenía una amistad que consumía frecuentemente alcohol. 
Chávez, Pertzelova y Zapata (2009: p. 149) demostraron la actitud desfavorable hacia la práctica abortiva como consecuencia de la primer relación sexual y el embarazo no deseado consecuente. Cerca del 26 por ciento de la muestra encuestada declaró usar un instrumento o dispositivo para prevenir el embarazo.

Fernández, et al. (2010: p. 320) encontraron que el rango de edad en el que más se practican abortos es del 19 por ciento para la muestra de entre 26 y 30 años. El 16 por ciento se ve obligada a abortar por la presión de la pareja, familia o su situación económica baja.

Serrano (2011: p. 219) demostró un incremento de los conocimientos sobre la prescripción abortiva de emergencia en relación con los años de residencia médica. En la medida en que el tiempo de residencia se incrementaba, se observaba un mayor porcentaje de conocimientos sobre la prescripción abortiva de emergencia.

En síntesis, la práctica abortiva inducida propicia diferencias significativas entre los factores socioeconómicos. Principalmente, entre rangos de edad, ingreso, escolaridad y estado civil. Se observa una tendencia de estudios actitudinales y psicológicos descriptivos en comparación a los estudios exploratorios o explicativos. El estado del conocimiento refleja una prevalencia descriptiva de las variables sociodemográficas en relación a los conocimientos, actitudes y comportamientos en torno al aborto inducido.

Sin embargo, el estado del arte no ha establecido las dimensiones actitudinales porque sólo se ha enfocado en su descripción comparativa con conocimientos y comportamientos en relación a los factores socio-demográficos asociados. Por ello, es menester explicar las dimensiones actitudinales en torno al aborto inducido para demostrar que la actitud no es sólo de orden cognitivo e intencional sino afectiva en torno a la cual, los individuos asocian la interrupción del embarazo con la moralidad y la religión. Dicha actitud afectiva, incluye emociones que determinan una actitud a favor del aborto si el embarazo es el resultado de una violación y una actitud en contra del aborto si el embarazo es atribuido a la irresponsabilidad de la mujer o de la pareja.

\section{Método}

El estudio fue de corte transversal en el que sólo se realizó un diagnóstico de las actitudes hacia el aborto inducido y se elaboró un estudio correlacional en el que se demostró la hipótesis en torno a la 
cual las actitudes hacia el aborto inducido están configuradas por indicadores evaluativos a favor y en contra de la práctica abortiva.

Muestra. Incluyó 300 universitarios. 149 son hombres y 151 mujeres. En cuanto a la edad; 86 (28.7 por ciento) tienen menos de 20 años, 137 (45.7 por ciento) entre 20 y 25 años, 63 (21 por ciento) entre 26 y 30 años y 14 más de 30 años. Respecto al nivel de estudio; 41 (13.7 por ciento) tienen bachillerato incompleto, 26 (8.7 por ciento) con bachillerato completo, 56 (18.7 por ciento) con licenciatura incompleta, 74 (24.7 por ciento con licenciatura completa, 17 (5.7 por ciento) con especialidad incompleta, 16 (5.3 por ciento) con especialidad completa, 25 (8.3 por ciento con maestría incompleta) y 45 (15 por ciento) con maestría completa.

Instrumentos. Escala de Actitudes hacia el Aborto Inducido el cual incluye 30 aseveraciones en torno a las evaluaciones favorables y desfavorables hacia la interrupción del embarazo, sus causas y consecuencias personales y familiares. Cada ítem fue evaluado a partir de una escala de siete puntos en la que -3 equivale a una evaluación "totalmente en desacuerdo" hasta +3 que significó "totalmente de acuerdo".

Procedimiento. A partir de las teorías y estudios actitudinales, se construyó una escala para medir las disposiciones favorables y desfavorables hacia el aborto inducido. Se utilizaron jueces para redactar y delimitar los 30 ítems a ocho. Una vez construida la escala, se procedió a su piloteo con una muestra intencional de 30 estudiantes de una universidad pública del Estado de Morelos. Posteriormente, se aplicó el instrumento a una muestra intencional de 300 universitarios de la facultad de psicología, administración y humanidades del campus Chamilpa-Cuernavaca de la Universidad Autónoma del Estado de Morelos. El criterio de elección fue presentar su credencial de estudiante y se procuró encuestar a las personas que acudieron a las bibliotecas de las facultades sin compañía. Una vez identificados los candidatos, se les invitó a contestar el cuestionario informándoles que tenían un tiempo límite de 15 minutos y al cabo de ellos se les recogería el cuestionario. Una vez revisadas las respuestas, se les invito escribir las razones por las que no contestaron algún ítem o utilizaron la misma opción en sus respuestas. Finalmente, se les agradeció su participación solicitándoles su correo electrónico para enviarles los resultados de la investigación.

\section{Resultados}


La tabla 1 muestra los valores de distribución normal en referencia a las medias de respuesta en cada ítem. Es posible observar que los valores de curtosis son negativos los cuales evidencia sesgos asimétricos en las respuestas a cada uno de los ítems.

\begin{tabular}{|c|c|c|c|c|}
\hline Reactivo & Media & Desviación & Curtosis & Factor \\
\hline $\begin{array}{l}\text { La familia que decide interrumpir un embarazo } \\
\text { protege el futuro de sus integrantes }\end{array}$ & 1.14 & .594 & .353 & .366 \\
\hline $\begin{array}{l}\text { El aborto inducido es una convicción que } \\
\text { afecta a la familia }\end{array}$ & 1.24 & .433 & -.172 & .368 \\
\hline $\begin{array}{l}\text { La decisión de interrumpir un embarazo es } \\
\text { una muestra de responsabilidad parental }\end{array}$ & 1.24 & .345 & -.161 & .468 \\
\hline $\begin{array}{l}\text { Los problemas económicos son suficiente } \\
\text { motivo para abortar }\end{array}$ & 1.90 & .672 & -.344 & .378 \\
\hline $\begin{array}{l}\text { La función familiar de una mujer depende de } \\
\text { sus responsabilidades como criadora de hijos }\end{array}$ & 1.05 & .422 & -.272 & .358 \\
\hline $\begin{array}{l}\text { Los padres que deciden abortar planifican el } \\
\text { futuro de su familia }\end{array}$ & 1.48 & .552 & .224 & .489 \\
\hline $\begin{array}{l}\text { Estudiar y tener hijos son decisiones } \\
\text { incompatibles para el futuro de las personas }\end{array}$ & 1.48 & .784 & -.275 & .318 \\
\hline $\begin{array}{l}\text { El aborto es una decisión particular de las } \\
\text { mujeres que sólo les interesa estudiar } \\
\text { Confialidad (alfa }=.768 \text { ) }\end{array}$ & 1.67 & .314 & -.147 & .343 \\
\hline Varianza explicada & & & & 56.7 \\
\hline
\end{tabular}

Tabla 1. Media, desviación, curtosis, confiabilidad y validez de las actitudes hacia el aborto inducido

Para subsanar el sesgo de asimetría, se procedió a efectuar una transformación monotónica consistente en multiplicar una constante por cada curtosis para sustraer el valor absoluto de las respuestas (Abad, Garrido, Olea y Ponsoda, 2006). Una vez establecida la normalidad, se llevó a cabo un análisis factorial exploratorio de componentes principales con rotación varimax. La tabla 1 muestra valores superiores a .300 que es mínimo requerido para incluir cada reactivo en el modelo reflejante de las actitudes hacia el aborto 
inducido. Posteriormente, se estimó la confiabilidad del instrumento a través del parámetro alfa de Crombach, la tabla 1 muestra un valor superior a .60 que es el mínimo requerido para establecer la consistencia interna de los ítems que incluyen el instrumento.

En torno al establecimiento de la estructura actitudinal hacia el aborto inducido, la matriz de covarianzas de la tabla 2 muestra los valores correlacionales entre los ítems que configuran la actitud había el aborto inducido. Pueden observarse valores tanto positivos como negativos entre los ítems a favor del aborto inducido y los ítems en contra de dicha actitud.

Tabla 2. Covarianzas entre los indicadores del factor actitudinal hacia el aborto inducido

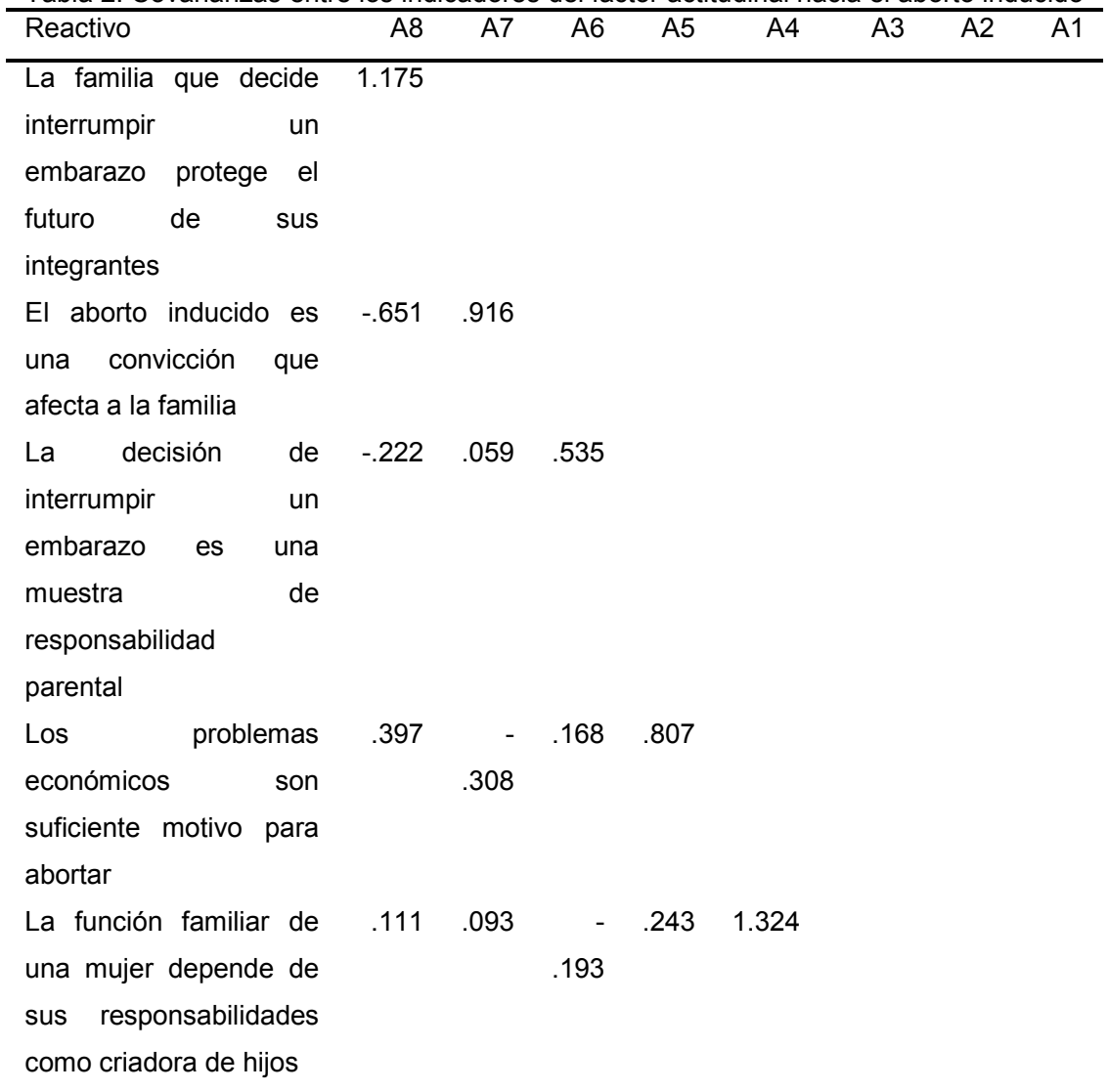




\begin{tabular}{|c|c|c|c|c|c|c|c|c|}
\hline Los padres que deciden & .508 & - & - & .274 & .023 & 1.039 & & \\
\hline $\begin{array}{l}\text { abortar planifican el } \\
\text { futuro de su familia }\end{array}$ & & .732 & .304 & & & & & \\
\hline $\begin{array}{l}\text { Estudiar y tener hijos } \\
\text { son decisiones } \\
\text { incompatibles para el } \\
\text { futuro de las personas }\end{array}$ & .257 & $\begin{array}{r}- \\
.101\end{array}$ & $\begin{array}{r}- \\
.212\end{array}$ & .068 & .508 & .219 & .678 & \\
\hline $\begin{array}{l}\text { El aborto es una } \\
\text { decisión particular de } \\
\text { las mujeres que sólo } \\
\text { les interesa estudiar }\end{array}$ & .127 & .087 & $\begin{array}{r}- \\
.254\end{array}$ & $\begin{array}{r}- \\
.214\end{array}$ & .341 & -.087 & .475 & .647 \\
\hline
\end{tabular}

Una vez establecidos los requerimientos para la estimación del modelo actitudinal, se llevó a cabo el cálculo de la estructura compuesta por una variable latente actitudinal y ocho indicadores. De acuerdo con las TRA, TPB y TCl, las actitudes son evaluaciones favorables o desfavorables sobre un entramado de situaciones en torno a un comportamiento, proceso, tecnología u objeto.

En el caso del aborto inducido, el estado del arte identifica a la presión familiar y a la situación económica como los determinantes de la toma de decisión abortiva. Ambos determinantes, sugieren que las actitudes son evaluaciones de las situaciones más que de las personas o el objeto mismo que este caso es el aborto inducido (López, et, al., 2001; Távara y Sacsa, 2008). Por ello, es fundamental establecer la estructura de las decisiones en torno al aborto inducido para esclarecer las causas reportadas por el estado del arte y su relación con otros indicadores sugeridos por el marco teórico.

Puesto que las evaluaciones, de acuerdo con las teorías actitudinales, suelen ser determinadas por las creencias, las percepciones y las situaciones. Se esperaría que los universitarios manejaran la información suficiente sobre el aborto inducido y que dicha información influyera en sus actitudes pro o anti aborto.

No obstante, puede observarse en la figura 1 que la propensión al futuro familiar, la responsabilidad y la planificación parental y los motivos económicos suponen evaluaciones deliberadas que coexisten con evaluaciones heurísticas de aversión prospectiva tales 
como la convicción anti-familiar, la incompatibilidad escolar-familiar y la crianza funcional. El modelo estructural explica el 32 por ciento de la varianza actitudinal lo cual sugiere la incidencia de otros indicadores no referidos por el estado del arte, la teoría y el presente estudio.

Figura 1. Estructura de la actitud hacia el aborto inducido 


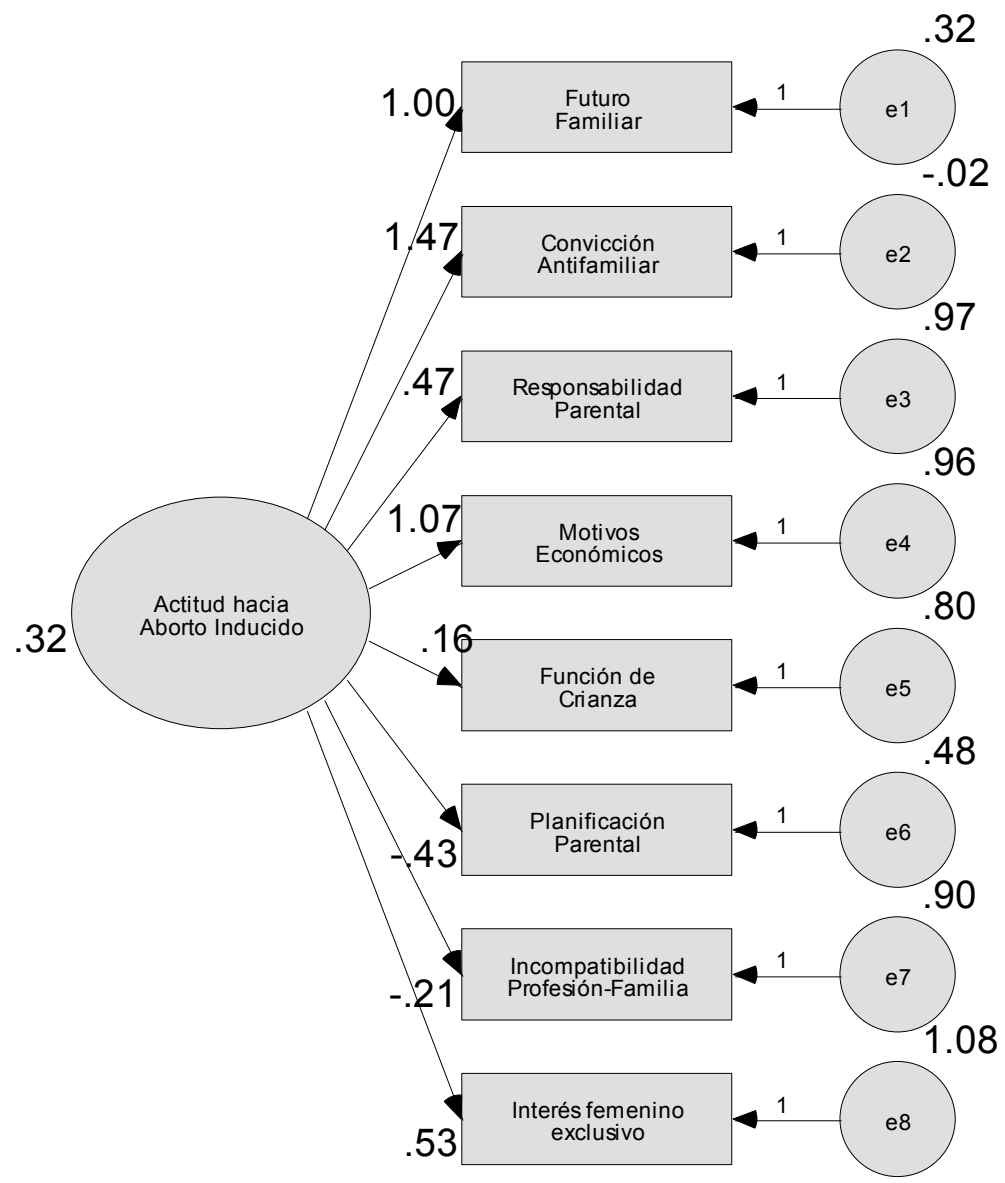

Sin embargo, el ajuste del modelo estructural en referencia al modelo de relaciones hipotéticas es cercano a la unidad lo cual se interpreta como adecuado. En el caso de los valores de ajuste residual, son cercanos al cero lo cual sustenta la hipótesis en torno a la cual la actitud es una estructura de evaluaciones a favor y en contra del aborto (ver tabla 3). 
Tabla 3. Índices de Ajuste de la estructura actitudinal hacia el aborto inducido

\begin{tabular}{|c|c|c|c|c|c|c|c|c|}
\hline Modelo & $\begin{array}{r}\text { Grado } \\
\text { s de } \\
\text { liberta } \\
d\end{array}$ & $\begin{array}{r}\text { Nivel de } \\
\text { significanci } \\
a\end{array}$ & $\begin{array}{r}\text { Chi } \\
\text { cuadrad } \\
a\end{array}$ & $\begin{array}{c}\mathrm{RM} \\
\mathrm{R}\end{array}$ & $\begin{array}{c}\text { RMSE } \\
\text { A }\end{array}$ & GFI & $\begin{array}{r}A G F \\
\end{array}$ & PGF \\
\hline $\begin{array}{l}\text { Actitud } \\
\text { pro- } \\
\text { abortiv } \\
\text { a }\end{array}$ & 20 & .000 & 19.753 & .018 & .023 & $\begin{array}{r}.91 \\
8\end{array}$ & .912 & .943 \\
\hline
\end{tabular}

Se trata de una ambivalencia en torno a la cual las evaluaciones pueden haber derivado de información a favor de planificación del futuro personal-familiar e información desfavorable al futuro puesto que inhibe la participación de la mujer en su desarrollo personal y familiar.

Ambas propensiones evaluativas, propensión al futuro y aversión prospectiva se discuten a continuación.

Conclusión

El presente estudio ha demostrado la estructura ambivalente de la actitud hacia el aborto inducido la cual incluye dos tendencias evaluativas: propensión al futuro y aversión prospectiva. Ambas dimensiones parecen coexistir al correlacionar positiva y negativamente entre sí.

En este sentido, la propensión al futuro consistiría en la inclusión, aceptación y participación de la mujer profesionista y ama de casa en la dinámica económica familiar para bienestar personal, interpersonal, parental y familiar. En contraste la aversión prospectiva sería la exclusión e incompatibilidad de la mujer como ama de casa, criadora de hijos y profesionista en la dinámica económica-familiar.

La propensión al futuro es una dimensión explicada por la TRA y TPB en tanto que la aversión prospectiva es objeto de estudio de la $\mathrm{TCl}$.

La propensión al futuro es un proceso latente en torno al cual las creencias, percepciones, actitudes, intenciones y comportamientos son guiados para delimitar un sistema de decisiones con miras al cumplimiento de objetivos que determinarían el razonamiento y la 
planificación por parte de los individuos en referencia a su vida futura sea interpersonal o familiar.

Por el contrario, la aversión prospectiva explica el proceso mediante el cual las personas deciden excluirse o excluir la participación de mujeres embarazadas que podrían obstaculizar el bienestar personal, interpersonal o familiar en el futuro.

Es decir, las causas y consecuencias del aborto inducido reportadas en el estado del arte parecen develar una actitud hacia el aborto relacionada con la situación personal-familiar actual y futura en la que la participación de la mujer parece ser o bien fundamental o bien intrascendente.

En este sentido, los estudios actitudinales podrían avanzar en la explicación de ambas dimensiones actitudinales a través de experimento que demuestren la ambivalencia actitudinal; la propensión al futuro y la aversión prospectiva. Esto supone la readecuación de las teorías, la inclusión de otras que expliquen la aversión prospectiva y la invención de otras que puedan explicar la ambivalencia actitudinal.

Dado que la tendencia económica-poblacional puede incrementar los casos de aborto inducido, esta problemática será el foco de atención de las políticas públicas. En torno a la salud y bienestar familiarpersonal, la agenda pública deberá incluir las causas actitudinales que activan las decisiones de planificación familiar o por el contrario aquellas actitudes que inciden en las decisiones de explosión demográfica las cuales vendrían a agravar la situación y sobre todo, el futuro de la humanidad, las regiones y las localidades que impedirán su desarrollo sostenido.

\section{Fuentes de Consulta}

ABAD, F., Garrido, J., Olea, J. y Ponsoda, V. (2006). Introducción a la psicometría. Teoría Clásica de los Test y Teoría de la Respuesta al ítem. Madrid: Universidad Autónoma de Madrid. 
- AJZEN, I. \& Fishbein, M. (1974). "Factors influencing intentions and the intention behavior relation". Human Relations. 27, 1-15

- AJZEN, I. (1991). "The Theory of Planned Behavior". Organizational Behavior and Human Decision Processes. 50, 179-211

- AJZEN, I. (2001). "Nature and operation of attitudes". Annual Review Psychology. 52, 27-58.

- BRIOZZO, L., Vidiella, G., Vidarte, B., Ferreiro, G., Pons, J., Cuadro, J. (2002). "El aborto provocado en condiciones de riesgo emergente sanitario en la mortalidad materna". Revista Médica de Uruguay. 18, 4-13

- CABEZAS, E., Langer, A., Luisa, A., Bustamante, P. (1998). "Perfil sociodemográfico del aborto inducido". Salud Pública de México. 40, 265-272

- CALDERÓN, J. y Alzamora, L. (2009). "Factores de pareja y grupales relacionados al aborto provocado en mujeres adolescentes". Scientia. 1, 110-116

- CHÁVEZ, M. y Zapata, J. (2009). "Actitudes respecto a la sexualidad en estudiantes universitarios". Enseñanza e Investigación en Psicología. 14, 137-151

- Comisión Nacional de Población (2009). Encuesta Nacional de la Dinámica Demográfica. México: CONAPO

- $\quad$ FERNÁNDEZ, M. Carrillo, F., Leal, M., Carrillo, I., Carrillo, E., Lozano, M., Fernández, S. y Pastor, L. (2010). "Una aproximación al aborto e inmigración. El valor socioeconómico de la vida humana". Cuadernos de Bioética. 3, 313-327

- GALVÁO, L., Díaz, J., Osis, M., Clark, S. y Ellerston, C. (2000). "Anticoncepción de emergencia: conocimientos, actitudes y prácticas de los Ginecobstretas". Perspectivas Internacionales en Planificación Familiar. Número especial. 15

- GARCÍA, S., Lara, D. y Goldman, L. (2003). "Conocimientos, actitudes y prácticas de los médicos mexicanos sobre el 
aborto: resultados de una encuesta nacional". Gaceta Médica Mexicana. 139, 91-104

- GONZÁLEZ, H. (2000). "Aspectos teóricos para el estudio sociodemográfico del embarazo adolescente". Revista Frontera Norte. 23, 65-85

- LARA, H. (1987). "Premisas socioculturales, planificación familiar y aborto". Revista Sonorense de Psicología. 1, 26-36

- LÓPEZ, F., Lázaro, G., Díaz, J., Campos, N., Heinrich, M. y Redondo, S. (2001). "Conocimientos y actitudes sobre anticoncepción de emergencia de los médicos de atención primaria en el área de salud". Medifam. 11, 441-448

- RAMíREZ, C. (2000). "Conocimientos y actitudes hacia la sexualidad en profesores de educación secundaria". Revista Sonorense de Psicología. 13, 47-54

- SALAZAR, H. (2007). "Conductas, conocimientos y actitudes de estudiantes adolescentes de zonas urbanas de las ciudades frente a los métodos anticonceptivos". Revista de Psicología Herediana. 2, 34-44

- SÁNCHEZ, G., Jiménez, F. y Merino, V. (1999). Escala de actitud sexual. Análisis estadístico de la adaptación castellana. VII Conferencia Internacional de Evaluación psicológica: Formas y Contextos. Braga, Portugal: Universidad do Minho.

- SERRANO, M. (2011). "Conocimiento, actitud y percepción de los médicos residentes de ginecología y obstetricia del ISSSTE acerca de la anticoncepción de emergencia". Revista de Especialidades médico Quirúrgica. 16, 215-220

- TAPIA, A., Villaseñor, M. y Nuño, B. (2008). "Conocimientos y actitudes hacia el uso de la anticoncepción de emergencia en jóvenes universitarios". Revista Médica del Instituto Mexicano del Seguro Social. 46, 33-41

- TÁVARA, L. y Sacsa, D. (2008). Conocimientos actitudes y prácticas de médicos ginecobstetras en relación al aborto. Lima: Promsex 